\title{
变弯度机翼准定常流动分离特性的实验研究
}

杨文超 ${ }^{\circledR}$ ，杨剑挺 ${ }^{\circledR}$ ，王进 ${ }^{\circledR}$ ，杨基明 ${ }^{\mathbb{Q}^{*}}$ ，徐庶民 ${ }^{\circledR}$ ，凌鹏 ${ }^{(2)}$

(1) 中国科学技术大学近代力学系, 合肥 230027 ;

(2) 中国科学技术大学工程与材料科学实验中心, 合肥 230027

*联系人, E-mail: jmyang@ustc.edu.cn

收稿日期: 2012-03-13; 接受日期: 2012-03-16; 网络出版日期: 2012-04-18

国家自然科学基金资助项目(批准号: 90816026)

\begin{abstract}
摘要研制了一种可实现弯度主动变形的机翼模型, 搭建了相关的风洞测力及流场显示实验平台. 低 速风洞测力实验揭示了机翼弯度连续准定常变形下的流动分离特性, 发现其与固定弯度下的流动分离 有着显著区别, 采用前缘和尾缘两种分离机制分析和解释了造成这种差异的原因. 相关的流场测量结果 验证了上述解释. 在流动分离条件下, 研究表明可变形飞行器外围流场结构不仅与当前时刻的飞行器外 形及来流条件有关，而且还和流场变化的历史过程相关.
\end{abstract}

关键词可变弯度机翼, 流动分离, 粒子成像测速, 空气动力学

PACS: 47.15.Cb, 47.32.Ff, 47.85.Gj

doi: $10.1360 / 132012-272$

随着材料技术及自动控制的发展，可适应多任 务、多飞行条件的智能可变形飞行器不仅仅是一个先 进的空气动力学概念, 更是成为了跨学科研究热点, 并受到越来越多的关注 ${ }^{[1]}$, 而气动特性的研究为这一 概念的最终实现搭建了指导性的框架. 可变形飞行 器的变形方式多种多样, 诸如变后掠, 变展弦比和变 翼面积等. 其中变弯度由于在气动效率及操控性能 方面的显著优势而受到了特别的关注. Szodruch 和 Hilbig $^{[2]}$ 指出, 变弯度机翼不但可以优化飞行包络线 从而进一步提升气动效率, 而且可以拓展抖振边界 并能显著提升飞行器的机动性能. 基于可变弯度机 翼在气动性能上的巨大潜力, 国内外近些年来开展 了一系列相关研究, 主要涉及变弯度机翼的结构设
计及定常气动特性. Gandhi 和 Anusonti-Inthra ${ }^{[3]}$ 研究 了可变弯度机翼的蒙皮结构, Vos 等人 ${ }^{[4,5]}$ 采用压电材 料研制了可以实现大幅弯度变形的机翼模型, 相关 的风洞测力实验表明, 可变弯度机翼具有独特的气 动优势并能有效提升飞行器机动性能.

此外, 可变弯度机翼可以改善低 Reynolds 数条 件下低速飞行器的失速特性. 低 Reynolds 数流动由 于其层流边界层难以抵抗逆压梯度很容易发生流动 分离, 因而微飞行器定常气动性能相比常规飞行器 有明显下降 ${ }^{[6]}$. 可变弯度机翼由于其不存在常规机 翼舵面偏转带来的不光滑奇点, 能最大限度地维持 附着流动, 从而明显改善了其流动分离特性 ${ }^{[7]}$. 刘烜 等人 ${ }^{[8]}$ 采用曲柄滑块驱动机构带动机翼蒙皮设计了

引用格式: 杨文超, 杨剑挺, 王进, 等. 变弯度机翼准定常流动分离特性的实验研究. 中国科学: 物理学 力学 天文学, 2012, 42: 531-537 Yang W C, Yang J T, Wang J, et al. Experimental investigation on the quasi-steady flow separation behaviors of a variable camber wing (in Chinese). Sci Sin-Phys Mech Astron, 2012, 42: 531-537, doi: 10.1360/132012-272 
一个可变弯度机翼并采用粒子成像测速技术(PIV)研 究了其定常流动分离特性, 他们的研究表明可变弯 度机翼能增强微型飞行器的机动性并改善失速特性. Munday 和 $\mathrm{Jacob}^{[9]}$ 的研究表明变弯度机翼表面的高频 振动可以有效抑制流动分离. 通过 PIV 技术, Gerontakos 和 Lee ${ }^{[10]}$ 研究了机翼尾缘向上或向下偏离 对于失速俯仰运动的 NACA0015 机翼周围速度及浴 量场的影响. Molkia 和 Breuer 的研究指出, 柔性薄膜 构成的机翼通过流固耦合作用能够推迟失速且提供 更好的气动性能 ${ }^{[11]}$. 以往关于可变弯度机翼的气动 特性研究往往侧重固定弯度下机翼的定常气动特性, 其连续弯度变形带来的准定常气动特性及变形路径 对于气动性能的影响则受到较少关注. 本文作者基 于气动力测量及烟线流场显示, 在这方面研究已经 取得初步进展 ${ }^{[12]}$. 鉴于 PIV 测量可以定量完整地反 映靠近机翼表面的流场, 由此可进一步使得针对可 变弯度机翼所特有的复杂流动机理的认识得以深入. 基于 PIV 及气动力测量, 本文重点探讨可变弯度机 翼的准定常气动特性以及变形路径对于气动特性的 影响。

\section{1 机翼模型及实验装置}

首先设计了一种可以进行连续光滑弯度变形的 机翼模型。该机翼模型未变形的基准形状为 NACA0015 标准翼型. 如图 1(a)所示, 机翼中主要承 受风载的 D 形结构从机翼前缘延伸至弦长 $30 \%$ 处. D 形结构以后的机翼部分拥有如图 1(b)所示的内部结 构因而可以实现变形. 这部分机翼内部含有 5 排沿弦 向分布的铰链结构单元. 这种铰链结构能够支撑柔 性蒙皮的气动外形并在保持机翼厚度的情况下实现 弯度变形. $\mathrm{D}$ 形结构内部安装的舵机在齿轮等传动机 构的帮助下，可以将机翼后部下表面的一部分柔性 蒙皮卷入到 D 形结构中, 从而使得机翼上下表面产 生面积差. 这种面积差让机翼变得不对称, 换句话说 具有一定弯度. D 形结构由丙烯酸有机材料构成, 柔 性蒙皮为聚丙烯塑料. 由驱动负载实现的机翼弯度 可有多种方式衡量, 主要包括机翼尾缘偏离距离和 等效襟翼偏转角. 在本文中采用前一种定义方法, 所 以用无量纲尾缘偏离距离 $d^{*}=d / c$ 来定义弯度, 在这里 $c$ 指机翼弦长, $d$ 指尾缘偏离距离, 如图 1(a)所示.

实验在中国科学技术大学低速风洞进行. 气动
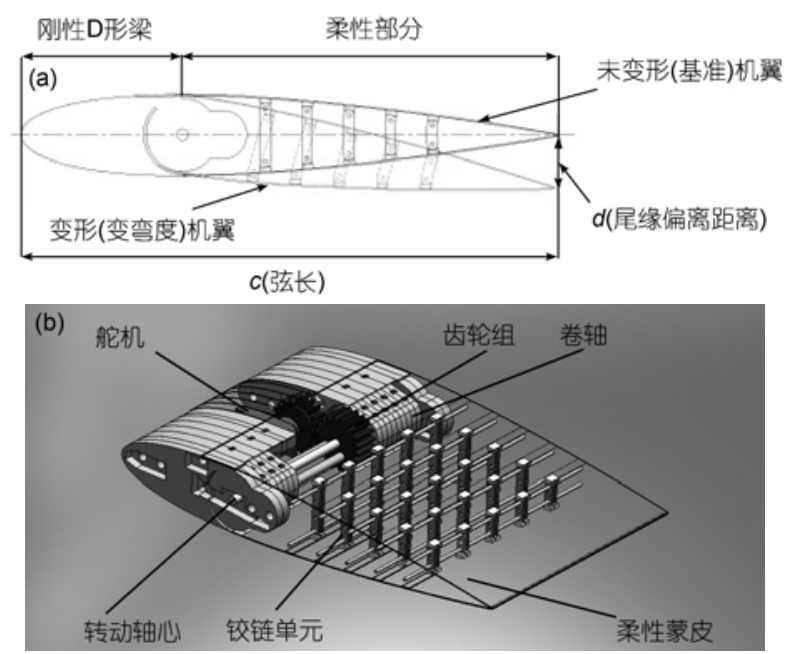

图 1 可变弯度机翼的结构

（a）基本设计; (b) 内部结构

Figure 1 Construction of variable camber airfoil. (a) Basic design; (b) internal structure.

力测量采用五分量测力天平. 可变弯度机翼弦长 200 $\mathrm{mm}$, 展长 $116 \mathrm{~mm}$. Reynolds 数 (以弦长为特征尺度) 固定在 $1.1 \times 10^{5}$, 因此风速设置在 $8 \mathrm{~m} / \mathrm{s}$ 并且通过热线 风速计进行监测. 机翼模型两端安装了直径 $30 \mathrm{~cm}$ 的 导流圆盘来抑制翼端三维效应.测力天平升力方向 精度为 $\pm 0.04 \mathrm{~N}$, 阻力方向精度为 $\pm 0.025 \mathrm{~N}$. 实验中同 时采用数码相机(CASIO EX-F1)来记录机翼的变形程 度. 测力平台的整体结构如图 2 所示.

流场显示采用粒子成像测速(PIV), 具体设备为 Dantec 公司的 Flowmap 1500. 示踪粒子采用液体石 蜡经烟雾机加热后的液体颗粒, 经反复实验发现此

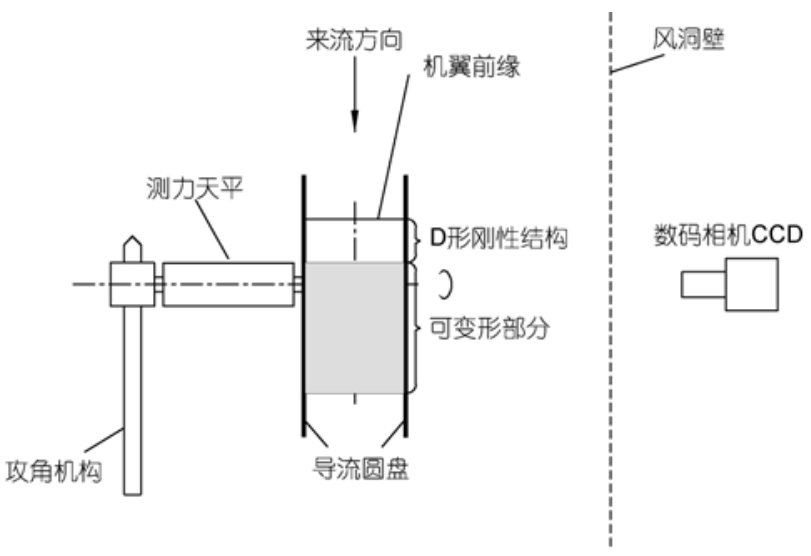

图 2 测力实验平台

Figure 2 Sketch of the aerodynamic load measurements setup. 
种颗粒不易粘附风洞壁面, 是理想的空气流场 PIV 示 踪粒子. $15 \mathrm{~Hz}$ 的双脉冲 $\mathrm{Nd}: \mathrm{YAG}$ 激光器(波长 $532 \mathrm{~nm}$, 脉冲宽度 $5 \mathrm{~ns}$, 能量 $500 \mathrm{~mJ}$, 两束脉冲间隔时间 $50 \mu \mathrm{s}$ ) 生成厚度大约 $1 \mathrm{~mm}$ 的片光照射翼型外围流场. 由于 流动分离只发生在翼型上表面, 所以片光只存在于 翼型上表面而下表面几乎全部被阴影覆盖，具体实 验平台如图 3 所示. 分辨率 1280×1024 的 CCD 记录

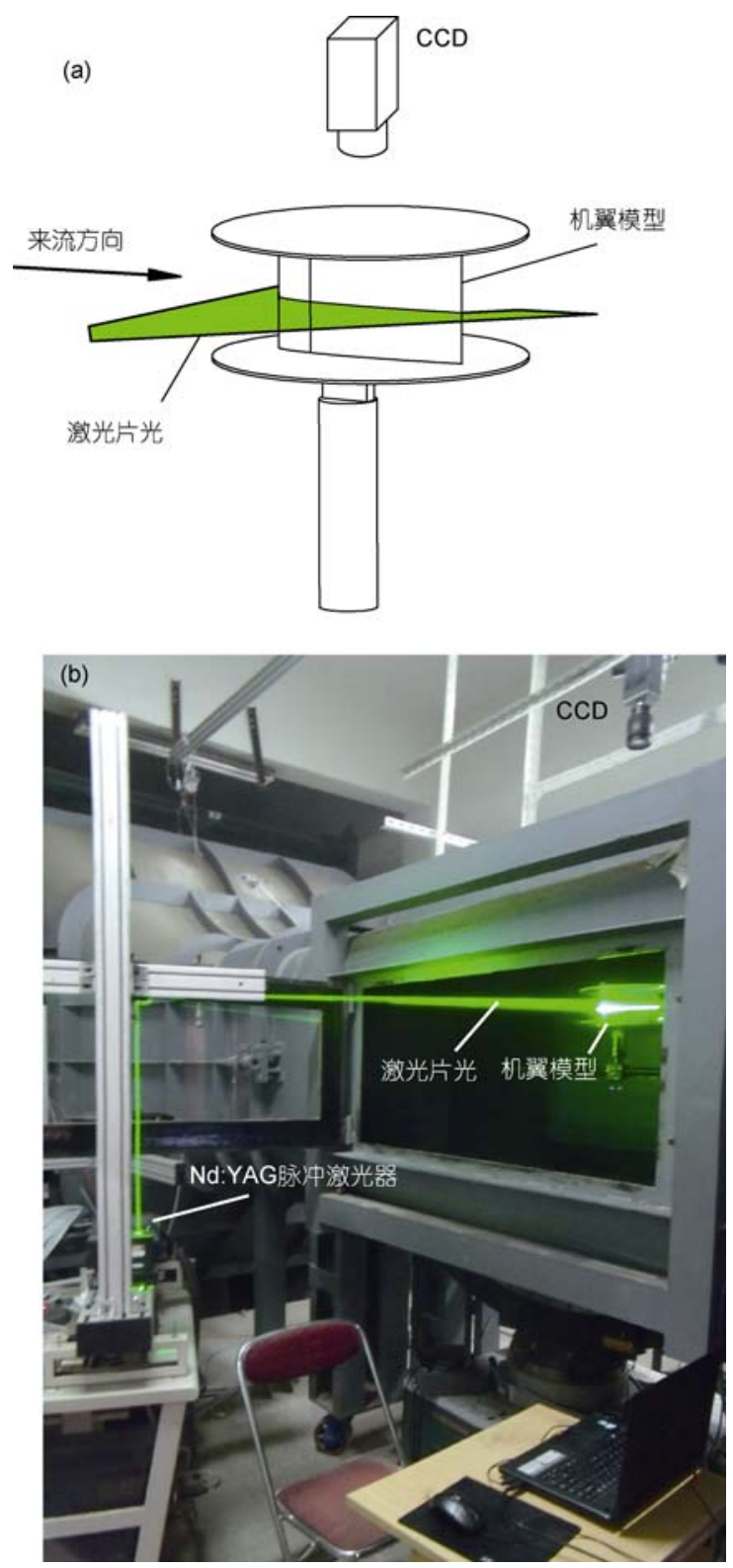

图 3 PIV 实验平台

(a) 原理图; (b) 实物图

Figure 3 Sketch of the PIV setup. (a) Schematic diagram; (b) real experimental setup.
$0.21 \mathrm{~m} \times 0.17 \mathrm{~m}$ 的物理空间, 即最小查询窗口(分辨率 16 像素 $\times 16$ 像素)的物理空间大小为 $0.0026 \mathrm{~m} \times 0.0026$ m. PIV 互相关计算采用剑桥大学的 Johan Kristian Sveen 编写的 MATLAB 程序 MatPIV V.1.6.1, 查询窗 口大小由像素 $64 \times 64$ 经 $32 \times 32$ 迭代至 $16 \times 16$, 每步计 算均采用 $50 \%$ 的重叠区. 互相关计算得到的速度场 经过滤波及平滑处理后得到最终结果. 这些后处理 方法进一步修正了流场中的非物理瑕疪，使得结果 更为合理可信.

\section{2 实验结果及分析}

为了对比机翼弯度准定常变化与定常弯度条件 下的气动力差异, 实验采用两种方法来测量升力系 数. 一种方式是静止条件下设置弯度在给定值, 然后 风速逐渐增加至给定 Reynolds 数, 待风速稳定后开 始气动力的测量. 在本文中这种测量被称为“静态测 量”. 另一种测量路径是在保持风速不变条件下, 逐 渐增加弯度, 同时依次测量各弯度下的气动力, 弯度 增加到最大值后再递减回起始弯度. 这种测量方式 称之为“连续测量”.

对于这两种测量, 攻角(AOA)均设置在最大升力 系数攻角 $\alpha_{l_{\max }}=16.5^{\circ}$. 大于此攻角的机翼很容易发生 失速，但该攻角下依然主要体现的是附着流动，这一 点可以从 PIV 得到的流场结构(图 4)中清楚地看到. 此时机翼上表面流向速率沿流动方向逐渐递减，可

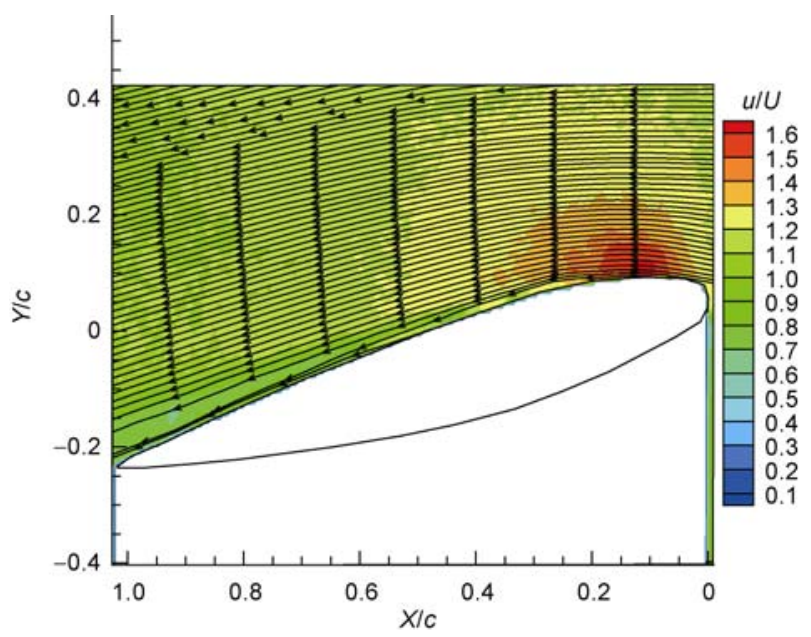

图 4 攻角 $16.5^{\circ}$, 无弯度条件下, 无量纲流向平均速度场 及流线

Figure 4 PIV results at 0 camber $(d / c=0)$ under $A O A$ of $16.5^{\circ}$. 
见存在明显的逆压梯度, 但是由于没有发生明显的 分离, 流场中并未出现剪切层和浴结构. 不过需要说 明, 此结果由多个时刻的 PIV 结果平均而成, 因而 “抹去”了流场的非定常脉动等微小差异, 体现出流 动的时间平均特性. 此外由图中可以观察到, 即使机 翼存在一定攻角, 其在水平方向的长度依然大于 20 $\mathrm{cm}$. 这是由于实验进行速度标定的平面位于翼型展 向的中部, 因而靠近 $\mathrm{CCD}$ 镜头的翼型端面在图片上 显现得更大, 但这并不影响我们对于整体流动特性 的判断.

首先通过静态测量来研究机翼弯度变形的静态 失速特征. 具体过程是在无风条件下设置弯度在给 定值, 然后风速逐渐加至给定实验 Reynolds 数, 流动 稳定后进行测量. 实验中改变弯度 $d / c$ 从 0 至 0.11 , 每 步步长 0.01. 如图 6 所示, 最大升力出现在无量纲偏 离距离 $d / c$ 到达 0.03 时, 此后升力系数急剧减小. 本 文更为关注的是连续测量, 其结果也示于图 6 , 以便 与静态测量进行比较分析. 为了研究准定常气动特 性和初始条件对于流动后续发展的影响, 实验采用 了 4 种连续测量路径: (1) 弯度 $d / c$ 在无风条件下设置 为 0 , 然后风速增加至指定 Reynolds 数. 风速稳定后 弯度 $d / c$ 逐渐由 0 增加至 0.13 后再恢复至 0 , 在每个 指定弯度处测量气动力; (2) 运动方式初始与路径(1) 相同, 但机翼的弯度 $d / c$ 经过增长阶段后固定在 0.13 并保持一分钟以上; (3) 弯度 $d / c$ 在无风条件下设置 为 0.07 , 然后风速增加至指定 Reynolds 数. 风速稳定 后弯度 $d / c$ 逐渐由 0.07 增加至 0.13 后再恢复至 0 , 在 每个指定弯度处测量气动力; (4) 弯度变形如同路径 (3)一样, 在 0.07 处启动, 但经过增长阶段后像路径 (2)一样固定在 0.13 . 以上这些路径参数在图 5 中具体 给出. 每步弯度 $d / c$ 变形量约 0.007 , 每次变形运动大 约经历 $0.5 \mathrm{~s}$, 随后保持该机翼弯度形状 $10 \mathrm{~s}$, 这种设 计是为了能反映准定常气动特性, 因而每步变形都 很微小, 且整个过程经历足够长的时间以尽量减小 变形运动速率的影响.

每个指定弯度下升力系数的平均值描述气动力 和弯度之间的关系. 如图 6 所示, 对于路径(1), 失速 弯度以前的升力系数曲线和静态测量得到结果很接 近. 然而路径(1)在弯度进一步增长后升力系数曲线 并未出现剧烈的下降, 而在最大升力附近表现出光 滑弯曲. Anderson 关于薄翼和厚翼气动力特性区别的 论述可以为上述现象找到类似的解释, 所不同的是

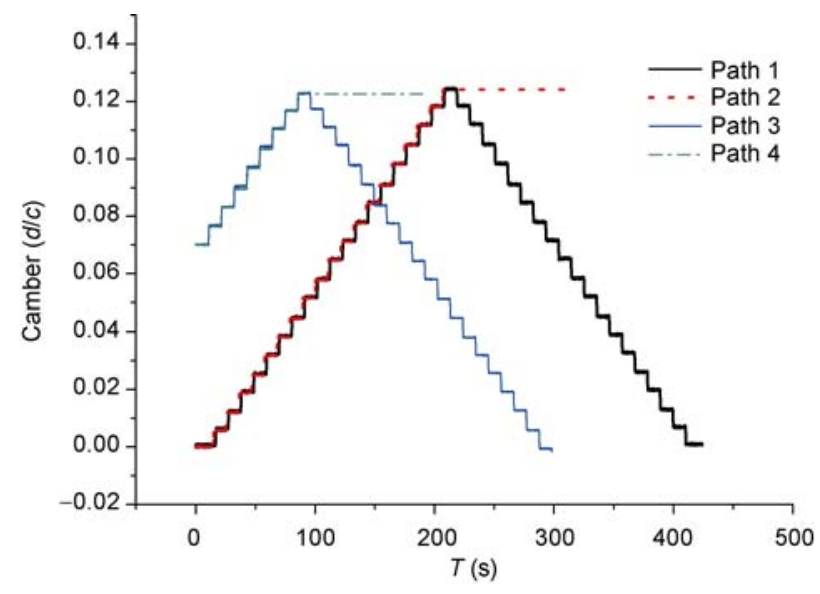

图 5 (网络版彩图)四种变形路径的弯度随时间变化规律

Figure 5 (Color online) Camber vs time for 4 Paths.

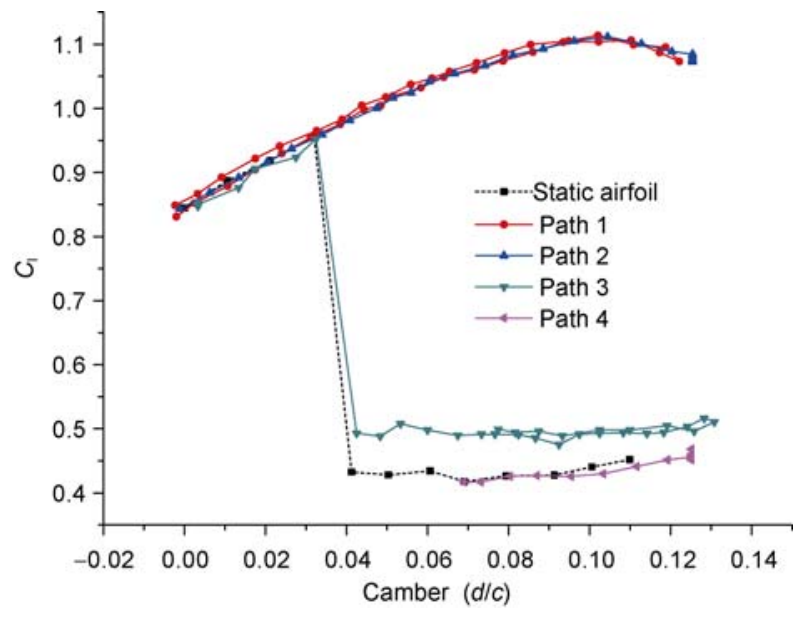

图 6 (网络版彩图)升力系数随弯度变化的规律

Figure 6 (Color online) Coefficient of lift vs camber.

控制变量在本文中由攻角变为了弯度 ${ }^{[13]}$. 基于这种 解释可以推断出两种分离特性决定了上述气动特性 的显著差别. 对于静态测量, 其失速特性是一种前缘 失速. 在这一失速过程中, 流动分离在整个机翼上表 面突然剧烈发生, 这种分离在机翼前缘就已经充分 发展. 因此如图 6 所示, 静态测量的升力系数曲线在 最大升力系数附近存在尖锐的极值点, 而跨过该点 (对应无量纲弯度 $d / c$ 接近 0.04 时)升力系数突然下降. 然而, 对于路径(1), 其失速是一种后缘失速. 这种失 速是随着机翼弯度的逐渐增加, 产生自尾缘的流动 分离逐渐由尾缘向前缘发展造成的. 因此这种尾缘 失速相比前缘失速更为柔和. 此外, 在路径(1)的升 力系数曲线中没有发现明显的迟滞现象, 对弯度增 
加或减小的过程路径似乎并不敏感. 这表明尾缘失 速由翼型后缘逐渐向上游发展的过程具有可逆的特 征. 如图 6 “Path 2"曲线所示, 路径(2)的升力系数曲 线和路径(1)极为相似, 因此可以看出路径(1)中当无 量纲弯度 $d / c$ 接近 0.13 时其气动特性为一种准定常特 性, 并未随着时间而较大范围波动. 路径(2)中, 在弯 度连续增加过程结束后, 保持该最大弯度状态至少 1 $\min$, 如图 6 所示, 路径(2)的数据在此位置呈现为一 组密集的点, 由此可以看出其气动力系数在此弯度 位置下保持稳定. 此时翼型表面的流场具有一段相 对稳定的附着流动以维持较高的升力, 而同样外形 下静态测量在升力系数方面则体现出明显的下降. 路 径(2)的结果表明静态测量与路径(1)在气动特性上的 差别并不来自变形的速率, 因为即便变形在最大弯 度处停止, 此时翼型的气动特性与正在变形过程中 的翼型并没有明显差别.

路径(3)中, 风速增加至指定 Reynolds 数后, 初 始弯度 $d / c=0.07$ 的机翼模型首先经历了前缘失速. 此 后在风速稳定的条件下, 机翼变形至最大弯度并且 最终恢复至对称翼. 如图 6 所示, 由于前缘失速, 机 翼在相当长的时间内保持了较差的气动特性, 直至 弯度小于静态失速弯度. 此外, 路径(3)中机翼由前 缘失速转换到附着流动的转变点也恰好和静态测量 得到的失速弯度吻合. 正如所预计的, 路径(4)同样 表现出和静态测量类似的前缘失速特征. 在最大弯 度处, 无论机翼保持该形状多久, 路径(4)的气动力 特性都表现出失速状态. 有意思的是, 尽管路径(2) 和路径(4)都在最大弯度处保持相当长时间且具有相 同的边界条件, 它们的气动特性却完全不同. 由此可 见，在这种流动分离条件下，流场结构不仅与当前流 场环境有关, 而且还和此前的演变历史相关, 因此, 在低 Reynolds 数飞行器设计中, 有必要考虑流场发 展历史过程的影响.

为了对上述前缘失速和后缘失速的机理有一个 更加直观的认识和定量的考核, 本文选取若干典型 的流动状态进行了较为细致地 PIV 速度场测量研究. 图 7(a)给出了路径(2)在最大弯度处的流场; 而图 7(b) 描述了相应弯度下路径(4)的流场. 从流场显示结果 我们可以清楚地看到前缘失速和尾缘失速的显著差 别, 尽管这两种流动现象具有同样的边界条件和机 翼形状. 对于路径(2)而言, 机翼由对称形状逐渐变 形至该弯度的过程中, 流动分离逐渐从后缘向前缘
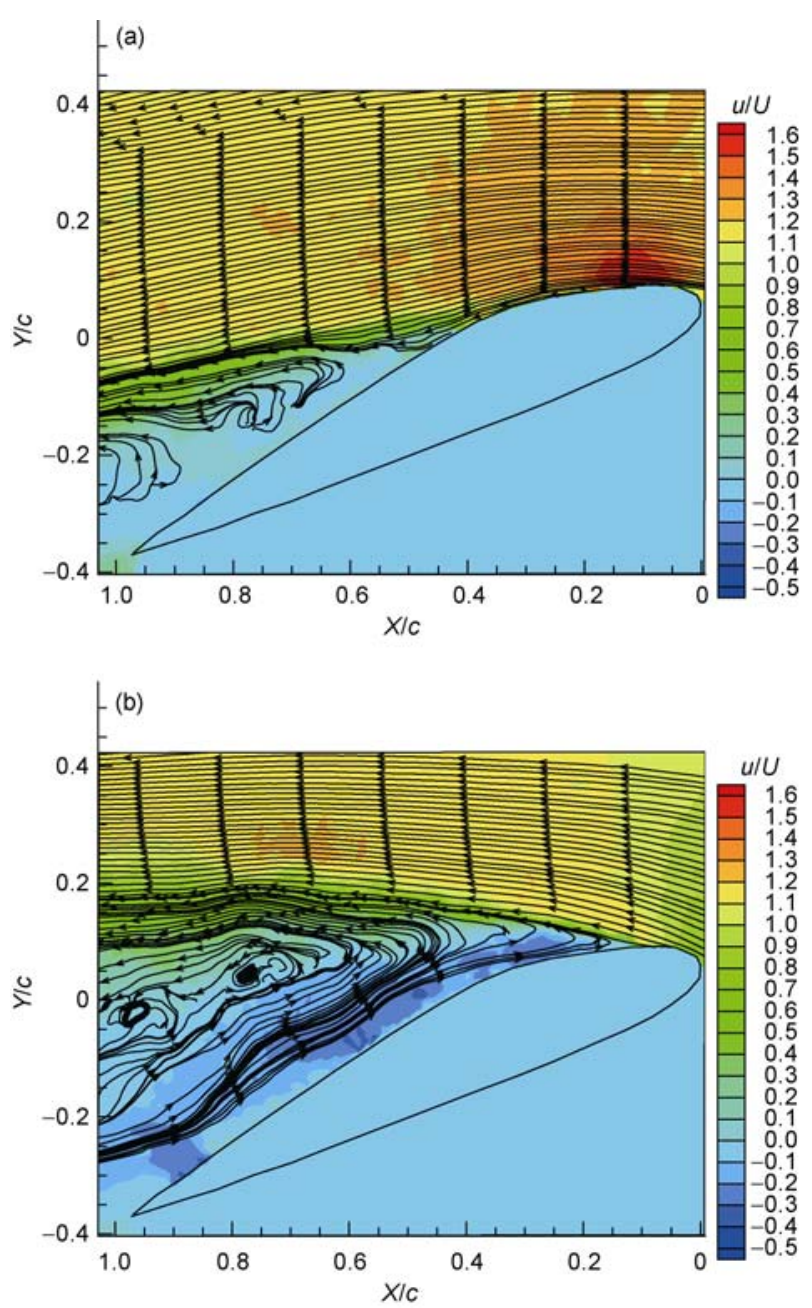

图 7 攻角 $16.5^{\circ}$, 最大弯度 $d / c=0.13$ 处的无量纲流向平均 速度场及流线

(a) Path (2); (b) Path (4)

Figure 7 PIV visualization results at maximum camber $(d / c=0.13)$ under $A O A$ of $16.5^{\circ}$. (a) Path 2; (b) Path 4.

发展. 从图 7(a)中可以看到在最大弯度 $d / c=0.13$ 处分 离点移动约至弦长的 $43 \%$ 处. 由于此时机翼上表面 前部依然处于附着流动且具有较强的负压区, 其升 力系数停留在较高水平. 图 7(a)中可以看到从机翼上 表面发展而来的剪切层将流场划分为外流和内部的 回流区. 由于剪切层的发展基本偏向 $-Y$ 方向, 将回 流区压缩在有限的空间内使其不影响到机翼前缘的 来流, 机翼外围的流动依然能够提供较大的升力.

与图 7(a) 明显不同的是, 图 7(b)描绘的路径(4)的 流场体现出显著的前缘失速特性. 在最大弯度 $d / c=$ 0.13 处, 分离点大约在弦长的 $8 \%$ 位置. 由于分离位 
置非常靠近翼型前缘, 翼型上表面没有出现明显的 负压区, 测量得到的升力系数较低. 此时剪切层从靠 近翼型前缘的位置生成且向 $Y$ 轴正方向发展, 从而划 分出了接近翼型尺度的回流区, 回流区内的流动速 度远远低于外流，导致翼型上表面没有存在明显的 负压区, 因而测量到的升力系数远远低于路径(2).

\section{3 结论}

通过风洞测力实验及相关的 PIV 流场显示, 机翼
弯度准定常变化所导致的流动分离特性表现出与定 常弯度条件下相应结果的显著差异. 这种差异是由 前缘失速和尾缘失速两种分离方式所决定的. 相同 的机翼弯度及边界条件下, 这两种分离方式都有可 能存在, 而不同分离方式的出现则与机翼变形过程 中先前时刻的流场环境及变形路径相关. 相关结果 表明: 对于可变形飞行器而言, 其外围流场结构可能 不仅与当前时刻的飞行器外形及边界条件有关, 而 且还和飞行器变形中流场变化的历史过程相关.

\section{参考文献}

1 崔尔杰，白鹏，杨基明. 智能变形飞行器的发展道路. 航空制造技术, 2007, (08): 38-41

2 Szodruch J, Hilbig R. Variable wing camber for transport aircraft. Prog Aerospace Sci, 1988, 25: 297-328

3 Gandhi F, Anusonti-Inthra P. Skin design studies for variable camber morphing airfoils. Smart Mater Struct, 2008, 17: 015025

4 Vos R, Barrett R, Breuker R, et al. Post-buckled precompressed elements: A new class of control actuators for morphing wing UAVs. Smart Mater Struct, 2007, 16: 919-926

5 Bilgen O, Kochersberger K, Inman D. Novel, bidirectional, variable-camber airfoil via macro-fiber composite actuators. J Aircraft, 2010, 47(1): 303-314

6 Mueller T J, DeLaurier J D. Aerodynamics of small vehicles. Annu Rev Fluid Mech, 2003, 35: 89-111

7 Chen Q, Bai P, Yin W L, et al. Analysis on the aerodynamic characteristics of variable camber airfoils with continuous smooth morphing trailing edge. Acta Aerodyn Sin, 2010, 28(1): 46-53 [陈钱, 白鹏, 尹维龙, 等. 可连续光滑偏转后缘的变弯度翼型气动特性分析. 空气 动力学学报, 2010, 28(1): 46-53]

8 Liu X, Zhu R, Liu J W, et al. Aerodynamics of an adaptive wing for MAVs (in Chinese). J Tsinghua Univ (Sci \& Tech), 2008, 48(5): 789-791 [刘烜, 朱荣, 刘敬威, 等. 用于微型飞行器的可变机翼设计及其特性. 清华大学学报(自然科学版), 2008, 48(5): 789-791]

9 Munday D, Jacob J. Active control of separation on a wing with oscillating camber. J Aircraft, 2002, 39(1): 187-189

10 Gerontakos P, Lee T. PIV study of flow around unsteady airfoil with dynamic trailing-edge flap deflection. Exp Fluids, 2010, 26: 339-358

11 Molkia M, Breuer K. Oscillatory motions of a prestrained compliant membrane caused by fluid-membrane interaction. J Fluids Struct, 2010, 26: $339-358$

12 Yang W C, Wang H, Yang J T, et al. Characterization of the flow separation of a variable camber airfoil. Chin Phys Lett, 2012, 29: 044701

13 Anderson J D. Fundamentals of Aerodynamics. Boston: McGraw-Hill Education, 2007 


\title{
Experimental investigation on the quasi-steady flow separation behaviors of a variable camber wing
}

\author{
YANG WenChao ${ }^{1}$, YANG JianTing ${ }^{1}$, WANG Jin ${ }^{1}$, YANG JiMing ${ }^{1 *}$, \\ XU ShuMin ${ }^{2} \&$ LING Peng ${ }^{2}$ \\ ${ }^{1}$ Department of Modern Mechanics, University of Science and Technology of China, Hefei 230027, China; \\ ${ }^{2}$ Experimental Center of Engineering and Material Sciences, University of Science and Technology of China, Hefei 230027, China
}

A test model of variable camber wing is developed as the first step of the work, which is capable of enough camber variation with acceptable mechanical stability and control accuracy. Corresponding experimental platform is established to conduct the aerodynamic load measurements and related flow visualization. Plentiful tunnel experiments depict distinction on the aerodynamic characteristics between the steady and quasi-steady flow separation behaviors. The distinction is interpreted with two types of stall mechanisms caused by the camber deformation: the leading-edge stall and the trailing-edge stall. Under the circumstance of flow separation, the morphing aircraft's flow structure depends not only on the current boundary conditions but also relates with the previous history.

variable camber wing, flow separation, particle image velocimetry (PIV), aerodynamics

PACS: 47.15.Cb, 47.32.Ff, 47.85.Gj

doi: $10.1360 / 132012-272$ 